\title{
APPLICATION OF SEISMIC GEOSTATISTICS TO IMPROVE THE TERRITORIAL INFRASTRUCTURE RESILIENCE AND SUSTAINABILITY
}

\author{
MASSIMO GUARASCIO, ANGELO LIBERTÀ, DAVIDE BERARDI, \\ ELEONORA DI BENEDETTO \& MARA LOMBARDI \\ Sapienza University of Rome, Italy
}

\begin{abstract}
The geostatistical methodology proposed in this article is able to provide quantitative data of the ground vibrational movements to the decision support systems to determine the priorities of the seismic interventions adaptation of existing transport infrastructures but also in the design phase of new infrastructures. This paper shows the use of geostatistics to estimate the arrival time and the value of the ground acceleration peak of the vertical and epicentral oscillation components of the Norcia earthquake of 2016. The validity of the non-stationary geostatistical methodology is confirmed by the calculated estimation errors in the 38 sites of the accelerometric stations and used to verify the representativeness of the estimated data. The histogram of the 38 normalized estimation errors is close to a Gaussian distribution with the frequency of the $\pm \sigma_{\mathrm{ck}}$ class of $73.7 \%$ instead of $68.3 \%$. This result was obtained for both seismic variables treated, arrival time of the maximum acceleration and peak ground acceleration value (PGA).

Keywords: geostatistical, seismic, resilience, sustainability, earthquake, seismicity, seismic hazard, historical earthquakes, seismic risk.
\end{abstract}

\section{INTRODUCTION}

\subsection{Regionalized geostatistical variables}

The knowledge with a low level of uncertainty of the directional seismic profiles of the ground oscillations following an earthquake is a relevant issue in active seismogenic areas. A uniform geographical coverage of quantitative seismic data is useful for decision support systems to determine the priorities of seismic adaptation interventions of existing transport infrastructures but also in the design phase of new infrastructures.

One response to engineering needs is the continuous monitoring of seismic quantities with an adequate geographical density of observation points to reduce as much as possible the level of uncertainty of the estimate in unobserved sites. At the same level of attention is the choice of efficient numerical methodologies for estimating the dynamic parameters of the vibrational movements of the ground, such as the Cartesian components of the amplitude, speed and acceleration vectors of the ground oscillations. This methodology should take into account the regionalized aspect of the seismic variables, strictly related to the geographic coordinates and the elevation of the site and not reducible to the epicentral distance alone.

The geostatistical methodology proposed in this article is able to provide the best local estimate of the seismic variables by making the most of existing data, but also to design seismic monitoring networks appropriate to the accepted degree of uncertainty. This paper shows the use of geostatistics to estimate at the nodes of a georeferenced grid the arrival time and the value of the ground acceleration peak of the vertical and epicentral oscillation components of the Norcia earthquake of 2016. 


\subsection{Seismic phenomenology and geostatistical model}

In the epicentral area of an earthquake, the vibrations of the ground layers of engineering interest are originated mainly from the seismic energy released in the hypocentral area and transported to the surface by longitudinal (primary waves) and transverse (secondary waves) volume waves. In the shallow earthquakes, with a hypocentral area between 7 and $10 \mathrm{~km}$ deep, the extension of the ground vibrations in the second following the instant in which the first volume waves reach the earth's surface is of the order of $10 \mathrm{~km}$. This is because in compact rocks the propagation velocity of volume seismic waves is between 3 and $6 \mathrm{~km} / \mathrm{s}$ and therefore the amplitude of seismic cone after about one second is about $30^{\circ}$.

The vibrational movement of the ground in the epicentral area generate new classes of seismic waves that propagate radially for hundreds of kilometers in the most superficial soil layer of geostatigraphic system. The amplitude of the vibrations of the ground outside the epicentral area is the resulting vector of the vibrational movements carried by the surface waves and the vibrational movements carried by the volume waves rising from the deeper layers of the geostratigraphic system. Moving away from the hypocentral area: (i) the amplitude of the surface waves of Rayleigh and Love become predominant with respect to the amplitude of the longitudinal (P) and transverse (S) volume waves; (ii) the dynamic parameters of the vibrational movements of the ground tend to weaken. Otherwise, in the innermost area of the epicentral area, the amplitudes of the vibrational movements carried by the volume waves, rising almost vertically from the hypocentral area, are stationary and do not have a precise and detectable zoning. In other words, the different damages suffered by the infrastructures of the road networks and by the houses of the urban centers located in this seismic area are attributable more to the different construction techniques than to a different seismic stress. A first prediction of the geographical pattern of the vibrational parameters of the ground such as amplitude, velocity and acceleration is provided by the physical theory of elastic waves applied to the waves emitted radially by a point source in a homogeneous and isotropic material medium. The theoretical result highlights a damping law of the dynamic parameters of the oscillations inversely proportional to the distance from the source for the volume waves $\left(\mathrm{f}(\mathrm{r})=\mathrm{a} \mathrm{r}^{-1}\right)$ and a law inversely proportional to the square root of the distance from the source for the waves superficial $\left(\mathrm{f}(\mathrm{r})=\mathrm{a}^{-0.5}\right)$ (geometrical attenuation).

In geostratigraphic systems, the regionalization of the vibrational parameters of the soil differs from the geographical pattern predicted by the theory due to the heterogeneity of the rock formations crossed by seismic waves and the complex geometry of the fault systems and the stratigraphic contacts between lithological units:

- the different propagation velocity of seismic waves in rock formations deform the geometry of the wave surfaces with consequent directional variations of the geometrical attenuation;

- moreover, irregular local variations of the seismic oscillations are a consequence of the different inelastic behaviour of the rock formations. A part of the energy carried by the seismic waves is continuously and irregularly dissipated by friction (anelastic attenuation).

Despite this, over distances of tens of kilometers, the data of the seismograms recorded by the accelerometric stations show good consistency with the geographic pattern described by the function $a r^{-0.5} e^{-b r}$ (first-guess); where $a$ is the adjustment factor to the existing seismic data; $r$ is the average distance from the epicentral zone of the maximum vibrations [1] and $b$ is the inelastic damping coefficient of the seismic oscillations. 
Fig. 1 shows the peak data of the epicentral component of ground acceleration (PGA) of the Norcia earthquake of 2016 and the attenuation profile predicted by the theoretical model. The graph highlights the presence of a directional anisotropy of the geographic pattern; the PGA data recorded by the accelerometric stations aligned in the $35^{\circ}$ East direction with respect to the epicentral area (direction orthogonal to the Apennine axis) are positioned in the lower band of the scatter-diagram. Directional anisotropy is also present in the vertical component of the PGA.

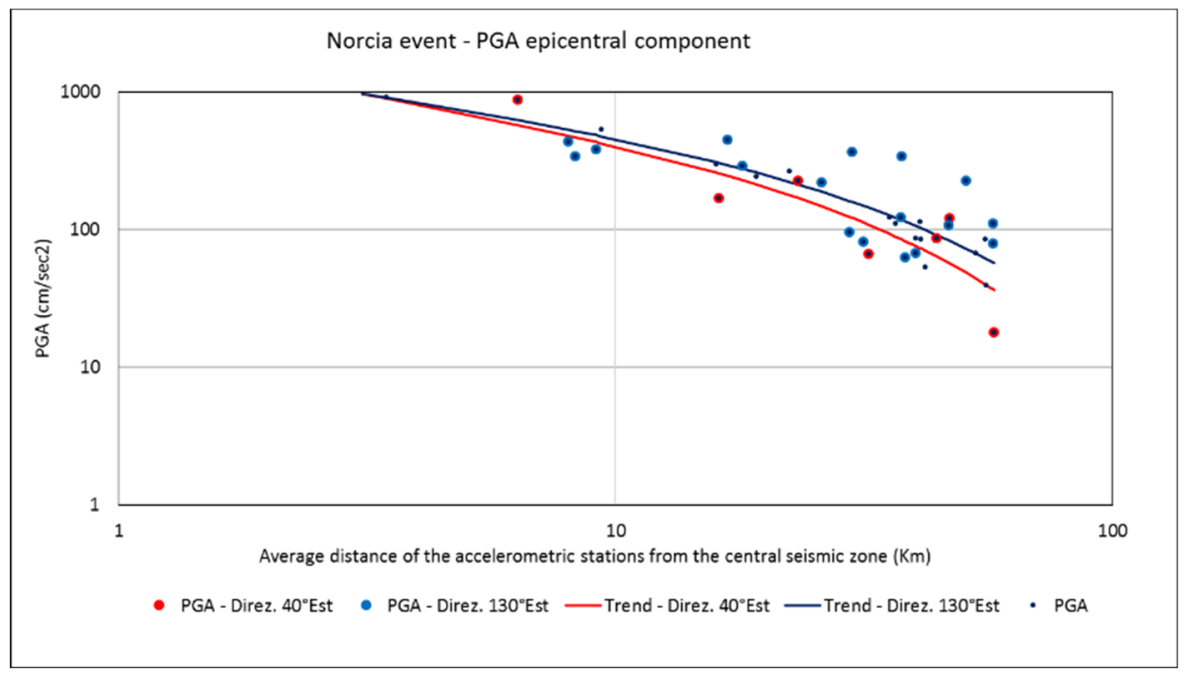

Figure 1: Theoretical model of geometric and inelastic damping of the acceleration peak vs PGA data recorded by the accelerometric stations during the Norcia earthquake (2016).

The goal of the work is to implement the best non-stationary linear estimator to estimate the arrival time and the local value of the acceleration peak at the nodes of a georeferenced grid with $500 \mathrm{~m}$ square cells. To this end it is:

- Assumed as a geographic pattern of the seismic variables: the function

$$
\begin{array}{ll}
a_{p} r^{-0.5}+e^{-b_{p} r} & \text { for the PGA } \\
a_{t}+b_{t} r & \text { for the arrival time of the acceleration peak }
\end{array}
$$

- $\quad$ used the Universal CoKriging geostatistical estimator with External Drift to integrate the functions of the geographic pattern of the seismic variables and the seismic information recorded by the network of accelerometric stations.

The geostatistical estimator, proposed as an alternative to the statistical methods already used to reconstruct the geographical evolution of the dynamic parameters of the vibrational movements of the ground (ground motions), allowed to: (i) reconstruct the directional attenuation profiles of the seismic variables consistent with the data and directional variations modelled on the data itself; (ii) calculate the local estimation error for any point of the observed geographic domain conditioned by the position of the accelerometric stations around the estimated point and the directional correlation of the seismic variables. In this way the estimation error or the uncertainty in associating the estimated value to a geographical 
point will depend not only on the distance from the epicentral zone [1]-[3] but also from the local and directional variations of the seismic variables. The validation phase of the geostatistical estimator has been added to the procedure for estimating the seismic variables. The aim is to check with the existing data: (i) the correctness of the estimator the average of the estimation errors is zero; (ii) the representativeness of the estimate uncertainty indicator the variance of the estimate calculated by the estimator is consistent with the variance of the estimation errors.

\section{GEOSTATISTICAL FRAMEWORK}

In this section, the principles of the geostatistical Universal CoKriging estimator are described and the definition of regionalized variable is briefly introduced.

\subsection{Natural phenomenon and regionalized variable}

Initially geostatistics was developed to estimate mineral deposits [4] but the same methodologies were found to be valid and therefore currently applied in many fields of earth sciences (hydrogeology, meteorology, oceanography, geochemistry, environmental control, soil science, etc.). The basis of geostatistical methodologies is the observation that some natural phenomena have a geographical evolution and local states are closely related to their position. This typology of phenomena have been defined as "regionalized phenomena" and consequently the variables that describe them are defined as "regionalized variables" (V.R.) [5]. The set of states assumed by a V.R. at points $x$ of a geographic domain they are represented by a function $\mathrm{z}(\mathrm{x})$. Locally, the $\mathrm{z}(\mathrm{x})$ function is highly irregular and the variations from one point to another are often unpredictable, while over greater distances the function is more continuous and shows the structural characteristics of the physical phenomenon that underlies the observed regionalized phenomenon. This feature suggested developing the function $\mathrm{z}(\mathrm{x})$ into a sum of two functions; the first, indicated by $\mathrm{m}(\mathrm{x})$, which represents the geographical trend of the regionalized phenomenon and the second, indicated by $\mathrm{y}(\mathrm{x})$, which reflects the irregular local variations of the observed phenomenon: $z(x)=m(x)+y(x)$. As they are defined, the two components are statistically independent. This double aspect is found in numerous geophysical and geochemical variables such as: temperature of the layer of atmosphere in contact with the earth's surface (temperature decreases with latitude and topographical altitude), concentration of pollutants in the soil (concentration increases near an oil plant) or vibrations of the ground following a seismic event (vibrations of the ground decrease with the distance from the epicentral area of the earthquake).

\subsection{Azimuth directional variography}

The irregular geographical evolution of natural phenomena has suggested the conceptual choice of considering the V.R. $\mathrm{z}(\mathrm{x})$ of the realizations of Random Functions (F.A.) Z(x) defined on a precise geographical domain $\mathrm{D}$ of the Euclidean space $\mathbb{R}^{n}$. The presence of a geographical pattern in some regionalized phenomena leads to the following model:

$$
Z(x)=m(x)+Y(x) .
$$

The function $\mathrm{m}(\mathrm{x})$ represents at each point $\mathrm{x}$ the expected value of the $\mathrm{F} . \mathrm{A} . \mathrm{Z}(\mathrm{x})$ : $\mathrm{E}|\mathrm{Z}(\mathrm{x})|=\mathrm{m}(\mathrm{x})$. The residual $\mathrm{Y}(\mathrm{x})$ of the $\mathrm{FA} \mathrm{Z}(\mathrm{x})$ is a locally stationary FA with $\mathrm{E}|\mathrm{Y}(\mathrm{x})|=0$ and covariance $\operatorname{Cov}\left|\mathrm{Y}\left(\mathrm{x}_{1}\right), \mathrm{Y}\left(\mathrm{x}_{2}\right)\right|=\mathrm{E}\left|\mathrm{Y}\left(\mathrm{x}_{1}\right) \mathrm{Y}\left(\mathrm{x}_{2}\right)\right|<\infty$. This model is generalized by assuming that the geographical variations of the FA $\mathrm{Y}(\mathrm{x})$ are stationary and depend only on the distance and direction (intrinsic hypothesis): 


$$
\gamma(\vec{h})=\frac{1}{2} \boldsymbol{E} \mid\left[\left(Y_{i}(x+\vec{h})-Y_{i}(x)\right]^{2} \mid .\right.
$$

The function $\gamma(\vec{h})$ is defined semivariogram of the F.A. stationary $\mathrm{Y}(\mathrm{x})$ and all the F.A. non-stationary defined as $\mathrm{Z}_{\mathrm{k}}(\mathrm{x})=\mathrm{m}_{\mathrm{k}}(\mathrm{x})+\mathrm{Y}(\mathrm{x})$ [5]. In the study of the natural phenomena described by several V.R. related, information on the geographical structure of the $F . A . Z_{i}(x)$ e $Z_{j}(x)$ is completed by the cross-variogram of the F.A. of the residuals $Y_{i}(x)$ and $Y_{j}(x)$ :

$$
\gamma_{i, j}(\vec{h})=\frac{1}{2} \boldsymbol{E} \mid\left[( Y _ { i } ( x + \vec { h } ) - Y _ { i } ( x ) ] \left[\left(Y_{j}(x+\vec{h})-Y_{j}(x)\right] \mid .\right.\right.
$$

The semivariogram or variogram and the experimental cross-variogram are calculated using existing observations from which the theoretical models are subsequently adjusted. This operation is done taking into account both the existing data and the phenomenological characteristics of the regionalized variables studied [6].

\subsection{Universal CoKriging with external drift}

Universal CoKriging with external drift is a specialization of Universal CoKriging (UCoK) applied when the functions that describe the geographic pattern of the regionalized variables studied (external drift) are known. UCoK is widely used in earth sciences to map geophysical or geochemical variables such as the minimum and maximum air temperature or the concentration of metals or pollutants in the soil. The UCoK estimator is numerically equivalent to a multivariate geographic regression conditional on the directional variogram and cross-variogram functions of the target variable and the auxiliary variables. The estimate of the target variable $Z_{0}$ at point $x$ is obtained from the linear combination (weighted average) of the data of the target variable $Z_{\mathrm{o}}$ and the auxiliary variables $Z_{i}$ which have a geographical correlation with the target variable:

$$
Z_{0}^{*}(x)=\sum_{k=0}^{m} \sum_{i=1}^{n} \lambda_{k, i} Z_{k}\left(x_{i}\right),
$$

where $\lambda_{0, i}$ are the weight coefficients of the target variable and $\lambda_{k, i}$ are the weight coefficients of the auxiliary variables (index $\mathrm{k}>0$ ) and $\mathrm{x}_{\mathrm{j}}$ are the coordinates of the $\mathrm{n}$ observation points $\left(\mathrm{x}_{\mathrm{j}}\right.$ represents the pair of geographic or metric coordinates necessary to locate the $i$ th point in the geographic domain).

The universal CoKriging with external drift assumes that (i) the average of the F.A. objective and the F.A. auxiliaries are in every point of the geographic domain equal to less than one or more multiplying factors to the functions that describe the respective geographic patterns, i.e. $E\left|Z_{i}(x)\right|=\sum_{l=0}^{L} a_{i, l} f_{i}^{l}(x)$; (ii) for each pair of points in the geographic domain, the mean of the squared differences of the F.A. and the average of the product of the difference between two F.A. are determined by the variogram and cross-variogram model:

For each point to be estimated the estimation coefficients $\lambda_{k, i}$ are obtained by setting the following conditions:

(i) average estimation error of the zero target variable: $\boldsymbol{E} \mid\left[\left(Z_{0}^{*}(x)-Z_{0}(x)\right] \mid=0\right.$. The condition of unbiased for the UCoK estimator becomes: $\lambda_{i}^{T} \boldsymbol{F}_{i, l}=\delta_{i, l} \boldsymbol{f}_{l}^{T}$

(ii) variance estimator error of the minimum target variable:

$$
\boldsymbol{V a r} \mid\left[\left(Z_{0}^{*}(x)-Z_{0}(x)\right] \mid=2 \sum_{i} \lambda_{i}^{T} \boldsymbol{\Gamma}_{i, 0}-\sum_{i} \sum_{j} \lambda_{i}^{T} \boldsymbol{\Gamma}_{i, j} \lambda_{j}=\min .\right.
$$

These two conditions are implemented in the following linear system [6]: 


$$
\begin{cases}\sum_{k=0}^{m}-\boldsymbol{\Gamma}_{i, j} \boldsymbol{\lambda}_{j}+\boldsymbol{F}_{i} \boldsymbol{\mu}_{i}=-\boldsymbol{\Gamma}_{i, 0} & i=0 \ldots m \\ \boldsymbol{F}_{i}^{T} \boldsymbol{\lambda}_{i}=\boldsymbol{f}_{l, 0} \delta_{i, l} & i=0 \ldots m\end{cases}
$$

where $\boldsymbol{\Gamma}_{i, j}$ are the nxn matrices of variograms or cross-variograms of F.A. $Z_{i}$ and $\mathrm{Z}_{j}$ between each observation points pair; $\boldsymbol{F}_{i, l}$ are the nxL matrix of the values of the first-guess functions of the V.R. $i$ th at the observation points; $\boldsymbol{\Gamma}_{i, 0}$ are the vectors of the variograms and crossvariograms between the point to be estimated (target-point) and the observation points of the F.A. to be estimated with the F.A. $Z_{i}$ and $\boldsymbol{f}_{l, 0} \delta_{i, l}$ are the vectors of the values of the firstguess function of the V.R. to be estimated and sold set to zero the conditions of unbiased of the V.R. auxiliaries.

The variance of the estimation error or cokriging variance is given by:

$$
\sigma_{C K}^{2}=\sum_{k=0}^{m} \lambda_{k}^{T} \boldsymbol{\Gamma}_{k, 0}+\mu_{l}^{T} \boldsymbol{f}_{i, 0},
$$

where $\boldsymbol{\lambda}_{i, 0}$ are the vectors of the F.A. $Z_{i}$ and $\boldsymbol{\mu}_{i, 0}$ are the vectors of the Lagrange parameters associated with the F.A. $Z_{i}$. The variance of the estimate quantifies the uncertainty of the estimate and, as defined, depends: (i) on the number of observation points; (ii) on the geometry of the observation points; (iii) on the position of the observation points with respect to the point to be estimated and (iv) on the local variability of the V.R. treated.

\section{NORCIA EARTHQUAKE OF 30 OCTOBER 2016}

This section presents the results of the seismic data processing of the 2016 Norcia earthquake (seismogenic zone of the Central Italian Apennines). The UCoK with External Drift estimator was used to estimate the time of arrival seismic variables and the peak value of the vertical $\left(\mathrm{PGA}_{\mathrm{V}}\right)$ and epicentral $\left(\mathrm{PGA}_{\mathrm{E}}\right)$ component of ground acceleration around $60 \mathrm{~km}$ from the epicentral area. The cartesian components peak values of the ground vibrational movements acceleration vector are some of the indicators most used in the design and seismic evaluation of road infrastructures and urban buildings [2], [7]. For this reason it was decided to test the UCoK with external drift geostatistical estimator on the two aforementioned seismic variables of the Norcia earthquake. To estimate the two seismic variables, the following were used: (i) the seismograms recorded by the accelerometric stations; (ii) the geographic coordinates and the quote of the accelerometric stations; (iii) the geo-referenced altimetric model with $500 \mathrm{~m}$ cells (DTM). The phases of the local estimation procedure of the seismic variables at the DTM nodes are:

A. localize the epicentral seismic area and the first-guess functions coefficients of the seismic variables;

B. determine the directional variogram of the seismic variables residuals not explained by the first-guess functions: difference between the real seismic data and the forecast provided by the theoretical model;

C. validate the geostatistical estimator using: the existing seismic data, the first-guess functions (point $\mathrm{A}$ ), the directional variogram (point $\mathrm{B}$ ) and the digital terrain model (DTM);

D. use the validated geostatistical estimator (point $\mathrm{C}$ ) to estimate the seismic variables at the nodes of the geo-referenced grid of the DTM.

\subsection{Ground motion data}

The two seismic variables data were determined from the seismograms of the vertical oscillation component and of the $\mathrm{N}-\mathrm{S}$ and $\mathrm{E}-\mathrm{W}$ oscillation components. To this end, the 
seismograms recorded by the accelerometric stations around $60 \mathrm{~km}$ of the geographical area with the largest number of buildings collapsed in the Norcia earthquake were examined.

In each accelerometer station the $\mathrm{PGA}_{\mathrm{V}}$ data are defined as follows:

$$
z_{v}(x)=\max \left[z_{v}^{\prime}(x, t) ; t_{0} \leq t \leq t_{f}\right],
$$

where $z^{\prime} k(x, t)$ are the acceleration data recorded in the vertical seismogram in the interval time between $t_{0}$ and $t_{f}$ referable to the seismic event. The arrival time of the vertical oscillations peak coincides with the instant $t$ of recording of the maximum data. The arrival time and the epicentral oscillations peak value on the epicentral seismogram produced by combining the data of the $\mathrm{N}-\mathrm{S}$ and $\mathrm{E}-\mathrm{W}$ seismograms were similarly determined.

Table 1: Quartile and max value of $\mathrm{PGA}_{\mathrm{V}}$ and $\mathrm{PGA}_{\mathrm{E}}$ (Norcia earthquake)

\begin{tabular}{|c|c|c|c|c|c|c|c|}
\hline \multicolumn{2}{|c|}{} & \multicolumn{2}{|c|}{ PGA vertical component } & \multicolumn{2}{c|}{ PGA epicentral component } \\
\hline Seismic event & $\begin{array}{c}\text { Number } \\
\text { of data }\end{array}$ & $\begin{array}{c}\text { I } \\
\text { Quartile } \\
\left(\mathrm{cm} / \mathrm{s}^{2}\right)\end{array}$ & $\begin{array}{c}\text { II } \\
\text { Quartile } \\
\left(\mathrm{cm} / \mathrm{sec}^{2}\right)\end{array}$ & $\begin{array}{c}\text { Max } \\
\text { value } \\
\left(\mathrm{cm} / \mathrm{s}^{2}\right)\end{array}$ & $\begin{array}{c}\text { I } \\
\text { Quartile } \\
\left(\mathrm{cm} / \mathrm{s}^{2}\right)\end{array}$ & $\begin{array}{c}\text { II } \\
\text { Quartile } \\
\left(\mathrm{cm} / \mathrm{s}^{2}\right)\end{array}$ & $\begin{array}{c}\text { Max } \\
\text { value } \\
\left(\mathrm{cm} / \mathrm{s}^{2}\right)\end{array}$ \\
\hline Norcia (2016) & 40 & 44.19 & 249.12 & 893.50 & 85.04 & 298.91 & 919.54 \\
\hline
\end{tabular}

\subsection{First-guess of seismic variables}

The first-guess fields of the two seismic variables are described by continuous mathematical functions defined at all points of the geographical survey domain:

Arrival time of the acceleration peak:

$$
m_{t}(x)=a_{t}\left(x_{0}\right)-b_{t}\left(x_{0}\right) \bar{r}_{0}(x),
$$

where $x_{0}$ identifies the coordinates pair of the center of gravity of the seismic event epicentral area; $a_{t}\left(x_{0}\right)$ and $b_{t}\left(x_{0}\right)$ are the coefficients of the regression line; $\bar{r}_{0}(x)$ is the average distance of the $\mathrm{x}$ coordinate point from the epicentral area (average of the distances between the point and the points of the circle with a radius of $5 \mathrm{Km}$ centred at the point $\mathrm{x}_{0}$ ).

Peak ground acceleration (PGA):

$$
m_{p}(x)=\frac{a_{p}\left(x_{0}\right)}{\sqrt{\bar{r}_{0}(x)}} e^{-b_{p}\left(x_{0}\right) \bar{r}_{0}(x)},
$$

where $x_{0}$ identifies the pair of coordinates of the center of gravity of the epicentral area of the seismic event; $a_{p}\left(x_{0}\right)$ and $b_{p}\left(x_{0}\right)$ are the coefficients of the theoretical pattern of the geographical evolution of the PGA; $\bar{r}_{0}(x)$ is, as for the seismic variable time of arrival, the average distance of the point of coordinates $\mathrm{x}$ from the epicentral area.

In the two first-guess functions the distance between any pair of points $\mathrm{x}^{\prime}$ and $\mathrm{x}^{\prime \prime}$ is defined as: $d\left(x^{\prime}, x^{\prime \prime}\right)=\sqrt[2]{\boldsymbol{X}^{T} \boldsymbol{H} \boldsymbol{X}}$ where $\boldsymbol{X}=\left(x_{1}^{\prime \prime}-x_{1}^{\prime}, x_{2}^{\prime \prime}-x_{2}^{\prime}\right)^{T}$ is the difference vector of the metric coordinates of the two points, and $\boldsymbol{H}=\boldsymbol{\psi} \boldsymbol{M} \boldsymbol{\psi}^{T}$ is the product of the rotation matrix of the cartesian reference system and of the metric tensor $\boldsymbol{M}$ used to introduce in the firstguess functions the directional anisotropy of the seismic variables:

$$
\boldsymbol{M}=\left|\begin{array}{cc}
(1+\alpha)^{2} & 0 \\
0 & 1
\end{array}\right|
$$


The coefficient $\alpha$ is the expansion factor of the metric coordinates of the Cartesian axis orthogonal to the Apennine alignment (oriented at $35^{\circ}$ East).

The position of the epicentral area must be compatible with the geographic pattern described by the $m_{p}(x)$ function and with the PGA data at the points of the accelerometric stations. To this end, the least squares estimator was first applied to estimate the coefficients of the first-guess function at the grid nodes of the DTM and then the grid node with the minimum root mean square error (RMSE) was selected as the centre of gravity of the epicentral area. In each node the error was calculated as the difference between the observed PGA data $\mathrm{z}_{\mathrm{p}}\left(\mathrm{x}_{\mathrm{i}}\right)$ and the value of the first-guess function at the point: $e_{p}\left(x_{i}\right)=z_{p}\left(x_{i}\right)-$ $m_{p}\left(x_{i}\right)$.

The estimate of the coefficients of the first-guess function of the $\mathrm{PGA}_{V}$ and the $\mathrm{PGA}_{\mathrm{E}}$ was obtained considering the quote $q(x)$ of the accelerometric stations and the grid nodes and assuming that the average of the F.A. $Z_{\mathrm{p}}(\mathrm{x})$ is expressed as:

$$
m_{p}(x)=c_{0}+c_{q} q(x)+\sum_{l=1}^{3} \frac{c_{l}(-1)^{l-1}}{\sqrt{\bar{r}_{0}(x)}} \frac{\left[\bar{r}_{0}(x)\right]^{l-1}}{(l-1) !},
$$

and solving three linear systems to calculate the estimation coefficients of $c_{l}^{*}(x)=$ $\sum_{i=1}^{n} \lambda_{l, i} z\left(x_{i}\right)$ :

$$
\left\{\begin{array}{l}
\boldsymbol{I} \lambda_{l}+\boldsymbol{F} \boldsymbol{\mu}=0 \\
\boldsymbol{F}^{T} \lambda_{l}=\delta_{k, l}
\end{array}\right.
$$

where $\boldsymbol{I}$ is the identity matrices $n \times n ; \boldsymbol{F}$ is the $n \times 5$ matrix of the values of the development terms of $\mathrm{m}_{\mathrm{p}}(\mathrm{x})$ in the observation points; $\boldsymbol{\mu}$ is the vector of the five Lagrange multipliers and $\delta_{k, l}$ is the operator to set equal to 1 the $k$ th term of the coefficient to be estimated. The coefficients of the first-guess function are calculated starting from the three coefficients $c_{l}^{*}(x)$. The coefficients of the first-guess time of arrival function are estimated by minimizing the error $e_{t}\left(x_{i}\right)=z_{t}\left(x_{i}\right)-a_{t}\left(x_{0}\right)-b_{t}\left(x_{0}\right) \bar{r}_{0}(x)$ on the $\mathrm{n}$ accelerometric stations.

\subsection{Seismic variables variography}

The variographic analysis of the two seismic variables of the vertical and epicentral oscillations of the Norcia earthquake was performed on the residual data calculated as the difference between the observed data and the value of the first-guess function at the point. For the seismic variable PGA it was observed that the variance of local dispersion of the residuals decreases with the distance from the epicentral area (Fig. 3), for this reason the experimental variograms and cross-variograms were calculated using the residuals of the time of arrival of the acceleration peak $y_{t}\left(x_{i}\right)=z_{t}\left(x_{i}\right)-m_{t}\left(x_{i}\right)$ and the normalized residuals of the PGA:

$$
y_{p}^{\prime}\left(x_{i}\right)=\frac{z_{P}\left(x_{i}\right)-m_{P}\left(x_{i}\right)}{\sigma_{P}\left(x_{i}\right)}
$$

where $\sigma_{P}\left(x_{i}\right)$ is the standard deviation of the residuals of PGA around the point $\mathrm{x}_{\mathrm{i}}$.

The structure analysis was performed by processing the pairs of accelerometric stations in the four directions: $35^{\circ}$ East (direction orthogonal to the Apennine alignment), $80^{\circ}$ East, $125^{\circ}$ East (direction of the Apennine alignment) and $170^{\circ}$ East. of accelerometric stations have been grouped in the four directions and for multiple lengths of $10 \mathrm{~km}$, applying respectively a directional tolerance of $\pm 20^{\circ}$ and a distance tolerance of $\pm 5 \mathrm{~km}$. For each 


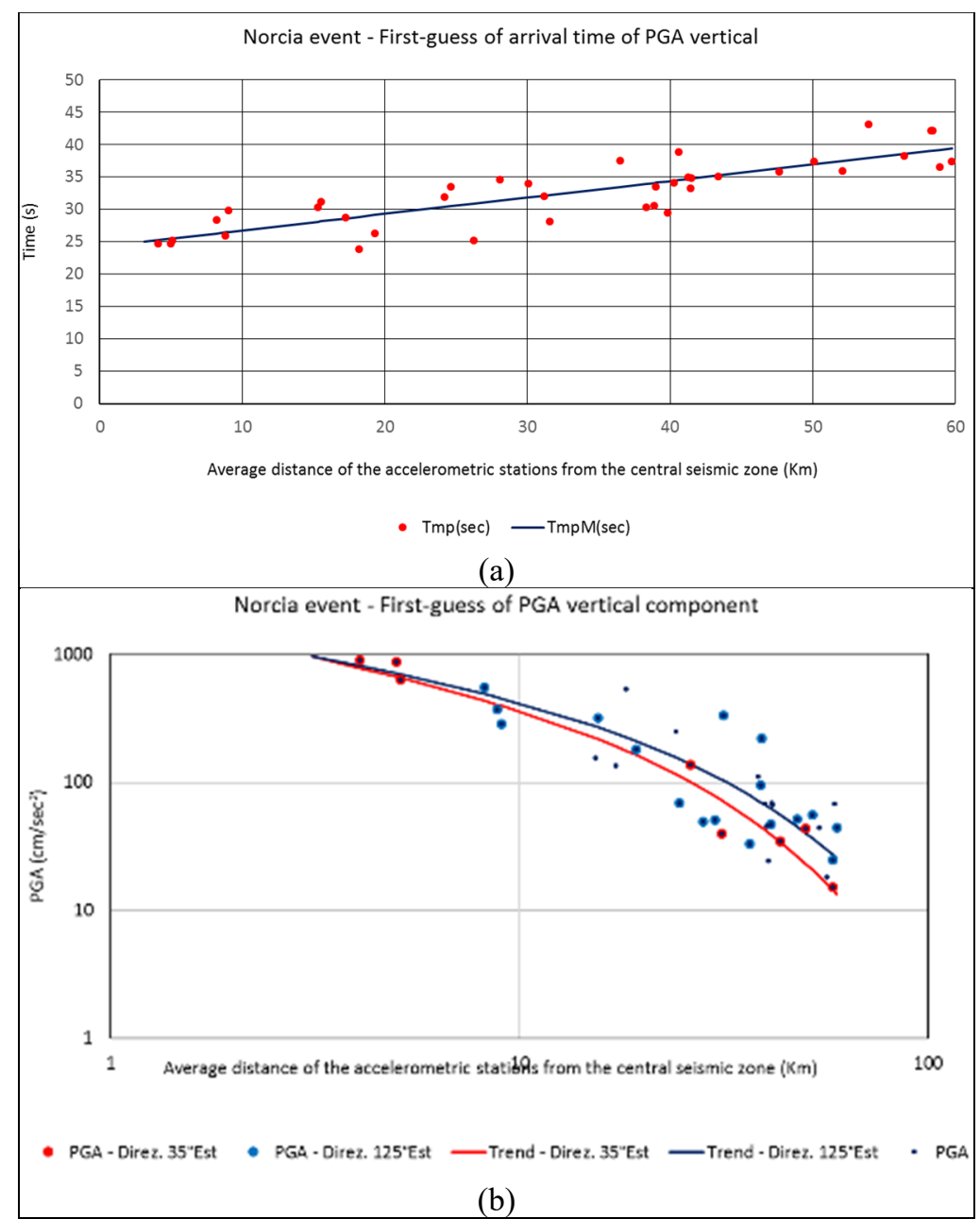

Figure 2: (a) First guess function of the arrival time vs (b) Vertical PGA during the Norcia earthquake (2016).

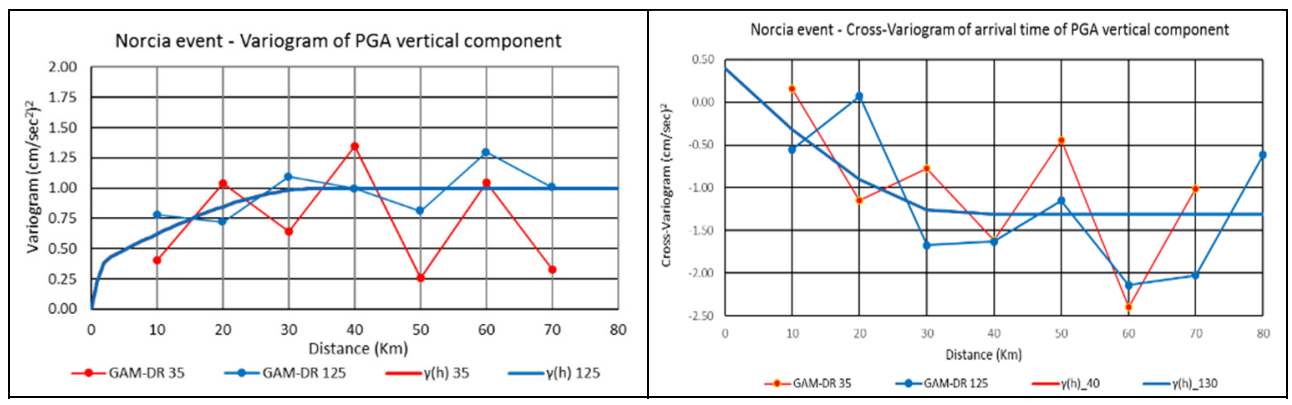

Figure 3: Variogram and cross-variogram of the peak value of the vertical acceleration with the arrival time of the Norcia event. 
direction-distance the variogram and the cross-variogram of the two seismic variables; for example, the PGA variogram of a direction-distance is calculated as:

$$
\gamma_{p}\left(h_{\alpha}\right)=\frac{1}{2 N\left(h_{\alpha}\right)} \sum\left[y_{p}^{\prime}\left(x+h_{\alpha}\right)-y_{p}^{\prime}(x)\right]^{2},
$$

where $N\left(h_{\alpha}\right)$ is the number of pair of accelerometric stations of the class classe $h_{\alpha}$.

The spherical scheme [6] defined by the following parameters was used to model the experimental variograms and cross-variograms: maximum correlation distance (range) and local dispersion variance. The variograms and the cross-variogram of the two seismic variables of the vertical oscillation components of the Norcia earthquake are shown in Fig. 3.

The variograms and the cross-variogram confirm the hypothesis of local stationarity of the two seismic variables of both the vertical and epicentral oscillation component. The variograms of the seismic variables of the vertical oscillation component detect the presence of a geographical correlation over distances of less than $30 \mathrm{~km}$. In particular, the geographical variations of the PGA are isotropic while for the arrival time of the acceleration peak there is a greater correlation in the direction $125^{\circ}$ East (Fig. 3).

For the epicentral oscillation component, the geographical correlation is detected only on the arrival time of the acceleration peak while the geographical variations of the PGA do not detect correlations over distances greater than $10 \mathrm{~km}$.

\subsection{Cross-validation}

Once the coefficients of the first-guess functions have been calculated and the variogram and cross-variogram models of the variables to be estimated have been chosen, the application of the estimation process is a sequence of numerical calculations well tested in practice.

Verifying the representativeness of these choices is therefore the phase to confirm the applicability of the estimator implemented to the project. For this purpose, the arrival time of the acceleration peak and the PGA were estimated at the sites of the accelerometric stations by applying the UCoK with external drift estimator and imposing on the local average of the two F.A. the following form:

- Arrival time of PGA: $m_{t}(x)=c_{t, 0}+c_{t, 1} \bar{r}_{0}(x)+c_{t, q} q(x)$

- $\quad$ PGA: $m_{p}(x)=c_{p, 0}+c_{p, 1} \frac{a_{p}^{*}}{\sqrt{\bar{r}_{0}(x)}} e^{-b a_{p}^{*} \bar{r}_{0}(x)}+c_{p, q} q(x)$

The estimates were made using the data of both seismic variables, but taking one as an auxiliary variable from time to time. The estimation process was applied to all the accelerometric stations, not considering each time the data of the station to be estimated. In this way, the estimation error of each was calculated as the difference between the real data and the estimated data and $\left(x_{i}\right)=z^{*}\left(x_{i}\right)-z\left(x_{i}\right)$.

The results reveal an estimation error very close to zero and a variance of the experimental error close to the estimation variance calculated by the applied estimator (Table 2). In the scatter-diagram of Fig. 4, the estimated data are compared to the detected data of the two seismic variables.

\section{ESTIMATION BY UNIVERSAL COKRIGING WITH EXTERNAL DRIFT}

The validated UCoK with external drift estimator was used to estimate the arrival time and the peak value of the vertical and epicentral components of the ground acceleration of the 
Table 2: Validation of the seismic variable estimation model (Norcia event).

\begin{tabular}{|c|c|c|c|c|c|c|c|}
\hline \multicolumn{2}{|c|}{} & \multicolumn{2}{|c|}{ PGA vertical component } & \multicolumn{3}{c|}{ PGA epicentral component } \\
\hline $\begin{array}{c}\text { Seismic } \\
\text { event }\end{array}$ & $\begin{array}{c}\text { Number } \\
\text { of data }\end{array}$ & $\begin{array}{c}\text { Average } \\
\text { errors }\end{array}$ & $\begin{array}{c}\text { Variance } \\
\text { errors }\end{array}$ & $\begin{array}{c}\text { Variance } \\
\text { estimate }\end{array}$ & $\begin{array}{c}\text { Average } \\
\text { errors }\end{array}$ & $\begin{array}{c}\text { Variance } \\
\text { errors }\end{array}$ & $\begin{array}{c}\text { Variance } \\
\text { estimate }\end{array}$ \\
\hline PGA & 38 & -0.52 & 7111.45 & 7026.15 & -0.60 & 10520.75 & 10835.20 \\
\hline $\begin{array}{c}\text { Arrival } \\
\text { time }\end{array}$ & 38 & 0.08 & 4.51 & 4.44 & 0.105 & 7.98 & 8.08 \\
\hline
\end{tabular}

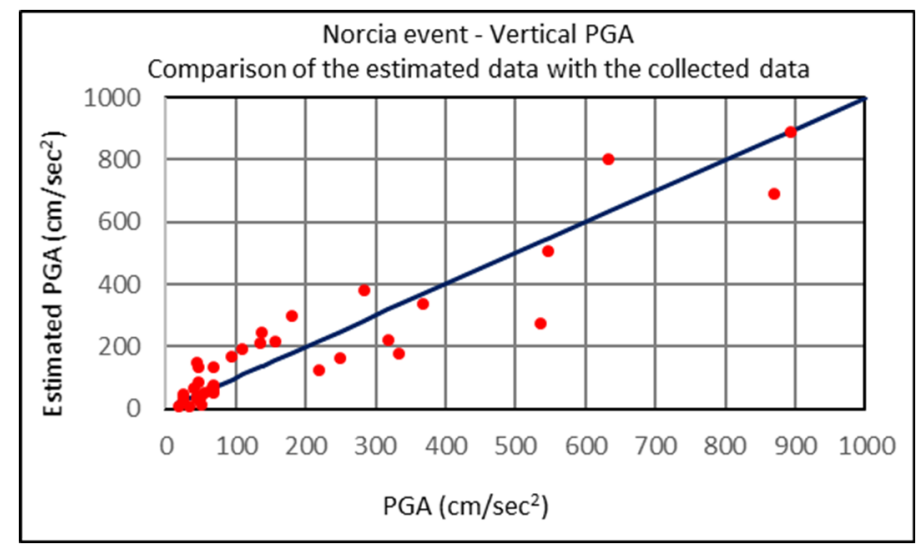

Figure 4: Cross-validation of the geostatistical model for estimating seismic variables.

Norcia earthquake (Fig. 5). The estimation was performed using data and first-guess functions of both seismic variables, variogram and cross-variogram models and digital terrain model (DTM). The value and variance of the two seismic variables were estimated in each node of the DTM grid.

\section{CONCLUSIONS}

The study highlights how the mapping of the estimated data represents the seismic phenomenology over the entire geographic domain. The local absence of observed data generated by a non-uniform distribution of the accelerometric stations is compensated by the physics law of damping of elastic oscillations in a homogeneous and isotropic material medium.

The network of observed data was used to adjust the physical law to the geostratigraphic system affected by the earthquake. This first result is representative of the geographical evolution of the two seismic variables over distances of tens of kilometers.

The non-stationary geostatistical estimator was applied to the data observed around each grid node of the DTM for a more precise evaluation of the local seismic state. The validity of the non-stationary geostatistical methodology is confirmed by the estimation errors calculated in the 38 sites of the accelerometric stations, which were used to verify the representativeness of the estimated data. The histogram of the 38 normalized estimation errors is close to a Gaussian with the frequency of the $\pm \sigma_{\mathrm{ck}}$ class of $73.7 \%$ instead of $68.3 \%$.

This result was obtained for both seismic variables treated: arrival time of the maximum acceleration and peak ground acceleration value (PGA). 


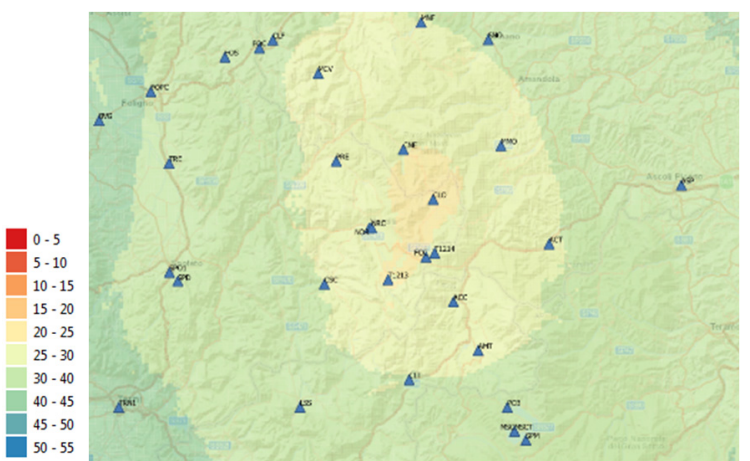

(a)

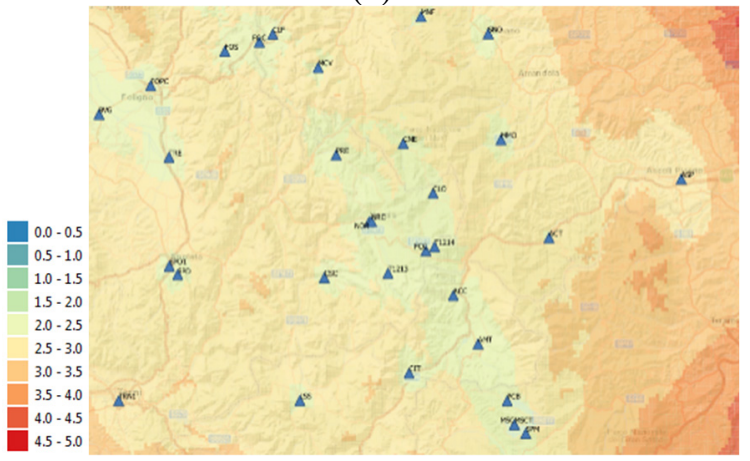

(b)

Figure 5: (a) Arrival time; and (b) Variance of estimation (Norcia earthquake).

\section{REFERENCES}

[1] Campbell, K.W., Strong motion attenuation relations: A ten-year perspective. Earthquake Spectra, 1(4), pp. 759-804, 1985. DOI: 10.1193/1.1585292.

[2] Sabetta, F. \& Pugliese, A., Estimation of response spectra and simulation of nonstationary earthquake ground motions. Bulletin of the Seismological Society of America, 86(2), pp. 337-352, 1996. DOI: 10.1785/BSSA0860020337.

[3] Hernandez, B., Cocco, M., Cotton, F., Stramondo, S., Scotti, O., Courboulex, F. \& Campillo, M., Rupture history of the 1997 Umbria-Marche (Central Italy) main shocks from the inversion of GPS, DInSAR and near field strong motion data. Ann. Geophys., 47(4), 2004. DOI: 10.4401/ag-3349.

[4] Guarascio, M., Huybrechts, C.J. \& David, M., Advanced geostatistics in the mining industry. Proceedings of the NATO Advanced Study Institute, Istituto di Geologia Applicata of the University of Rome, Italy, 13-25 October, 1975.

[5] Matheron, G., Random sets theory and its applications to stereology. Journal of Microscopy, 95, pp. 15-23, 1972. DOI: 10.1111/j.1365-2818.1972.tb03708.x.

[6] Chilés, J. \& Delfiner, P., Geostatistics: Modeling Spatial Uncertainty, Wiley InterScience Publication, New York, 2012.

[7] Guarascio, M., Lombardi, M., Rossi, G. \& Sciarra, G., Geostatistics/reliability based risk analysis of the Vajont landslide, pp. 607-615, 2009. DOI: 10.2495/SAFE090561. 